\title{
Survey of the Evolution of Two Points Belonging to Two Objects Falling without Initial Speed Dynamic Chart (Dot per Dot) Video (Frame by Frame) Coupling
}

\author{
Michel CALMET \\ University of Montpellier, University of Aix-Marseille
}

\begin{abstract}
*Corresponding author: Michel CALMET, University of Montpellier, University of Aix-Marseille, France; Email: michel.calmet@umontpellier.fr
\end{abstract}

Article Type: Research, Submission Date: 25 May 2016, Accepted Date: 26 August 2016, Published Date: 14 September 2016.

Citation: Michel CALMET (2016) Survey of the Evolution of Two Points Belonging to Two Objects Falling without Initial Speed Dynamic Chart (Dot per Dot) Video (Frame by Frame) Coupling. J Robot Mech Eng Resr 1(4): 1-9.

Copyright: () 2016 Michel CALMET. This is an open-access article distributed under the terms of the Creative Commons Attribution License, which permits unrestricted use, distribution, and reproduction in any medium, provided the original author and source are credited.

\section{Abstract}

This paper presents one experiment on a computer screen with a "dynamic chart" linked to a "video footage". As the user can shift dots in the chart simultaneously with the video footage playing frame by frame, this application is named: "chart-video coupling". Used in a physics experiment concerning gravity, this paper focuses on a possibility to model and to simulate in specific cases, when objects fall, one part of these objects can fall faster or slower than their centers of mass. This chart-video coupling was first elaborated for sport science students at the University of Montpellier. The use and the elaboration of this application about video motion analysis concern athletes, sports coaches, medical doctors and students. To train these in "ICT and sports", a course is organized at the faculty of sportscience at the University of Montpellier. Students have to choose and to build 4 applications among 5 . These applications are consistent with the certificates concerning computer sciences and Internet delivered by French Ministry of Higher Education and Research. These applications allow both training and research and put in evidence some events that human eyes cannot see easily. Satisfaction surveys have shown that students found this course adapted to their curriculum and it helps them to understand how professional software runs. Since 2007, applications have been updated and upgraded, and used by 6 teachers each year. They allowed 3 publications in sports totaling 92 quotes. These applications allow interdisciplinary approaches between ICT and physics. Examples were built about gravity, Newton's pendulum, and juggling. The one concerning gravity was used in adult education within Algerian universities. Perspectives are to build 3 dimensional graphs with two cameras and to integrate new applications on smartphone (accelerometers, gyroscopes).

\section{Introduction}

This paper presents, a way to show one experiment on computer

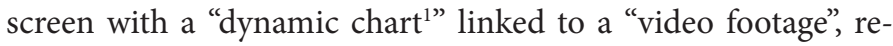

\footnotetext{
${ }^{1}$ To realize a dynamic chart, one can create two curves in a scatter chart:

1. the first curve is made with all data

2. the second curve is made with only one dot, belonging to the
}

spectively: "emergence of the key points to hold" and "complexity of the reality".

As the user can simultaneously shift dots in the chart and plays video footage frame by frame, this way is named: "chart-video coupling".

This chart-video coupling was first elaborated for sport science students and was applied to researches in sports skills analysis. In the faculty of sport science in the University of Montpellier, information and communication technologies (ICT) are taught and based on sports skills analysis. This is somehow a "continuity" with the works in sports initiated by Muybridge; Marey \& Demeny at the end of XIX ${ }^{\circ}$. These authors' works are described on recent books, respectively [1-3].

ICT can control image banks stored on external devices or on hard drives. Connected objects (some have existed at the end of XIX [4]) about sport activity and sports are numerous and allow observations or data collection that were inconceivable in recent years: analysis before training, helps during training but also speedometer, accelerometer, gyroscopes. Softwares to analyze video sequences in sport are numerous, but before 1996, microprocessors Intel Pentium MMX and CDROM x 6 $(900 \mathrm{KiB} / \mathrm{s})$, it was difficult to use computer and digitized video footage.

Video motion analysis is a technique who concerns different domains: sports, physics, speech or debate analysis. In June 2016, request with "video analysis in sport" in a web search engine gives back 64.800 .000 results. Among these results, 3 great categories:

Software about "video analysis in sport" (27.800.000 results)

- Features are: usable indoor, stadium, or in laboratory; running on different operating systems; usable in real time (Sportscode) or delayed time; some are customizable (Nacsport allows 25 buttons/criteria); enhanced video (key frame; tag on frames,

data therefore to the curve.

Shifting the dot with a scrollbar, step by step (i.e.: value 1 , value 2 , value $3 \ldots$ and so on) creates an animation in the chart: the dot seems follow the curve"dot per dot". Video footage as examples, show it. 
added chronometers, scales, draws, angles...); export data to XML / XLS files.

- They concern: all sports (team performance analysisor technique analysis); specific sport (golf, bowling, base-ball, tennis...) or health / fitness (heart rate, speed shifts, testing ...).

- Pricing: some are open source (Kinovea), some are free (Longo Match), almost all have one free trial (usually 30 days), and some are running on different operating system (Hudl with Android and iOS). One can buy software, pricing scale is: $€ 100$ to $€ 3000$ with free updates for 12 months or one can rent software $€ 500$ per year.

Benefits of video analysis in sports

- Improving athletes and teams' skills, improving coaches' analysis

Scientific papers (more than 480.000 results)

- Features are: Biomechanics, sports techniques, physics, sports and physics

Some softwares are customizable (user can create some buttons concerning criteria without really set the software), possibilities to customize are increasing according to the price. Benefits concern athletes, teams and coaches.

Scientific papers confirm three ways to use video analysis:

1) Before (video analysis allows "mistake" or replay to learn): to prepare or to train

2)During (in real time): to help, to improve performance or to assist

3) After (debriefing): to analyze, to understand, to confirm hypothesis

Athletes, sports coaches, medical doctor, teachers are concerned by the use and by the elaboration of these softwares. They have to expertise injuries, diseases, pattern analysis, statistics, specific sports algorithms, experiments.

If papers concern teaching or training session (training in one sport domain or training how to use a specific software), we didn't find paper concerning the possibility to train in ICT while learning the basic knowledge of a video analysis application. To train students in ICT and video analysis we built a course "ICT and sports" in which students have to choose and realize, following a pedagogical method as "La main à la pâte" [5], 4 applications among 5 .

\section{Aims}

These applications allow both training and research:

1) Students in sports sciences improve their ICT knowledge and refine their video analysis

2) Teachers can collect and analyze data to allow scientific publications.

\section{Information concerning the course}

The course was first ended in 2007/2008, it is regularly updated/ upgraded and it contains tutorials that allow to build applications including the chart-video coupling. Applications are built with spread sheets (MS-Excel) and videos are recorded with digital cameras (30 f/s to $210 \mathrm{f} / \mathrm{s})$.
The ICT course contents are consistent with certificates concerning computer sciences and Internet delivered by the French Ministry of Higher Education and Research: C2I [6] (level1: bachelor) and C2I2E [7] (level 2: master).

To reach the objectives targeted in ICT and in analysis of sports activities, several software and hardware are mainly used:

1. Kinovea [8] as free software for video analysis: it allows to index the videos (key images and comments), it allows to add stopwatches, tools to measure and realize some post-production.

2. Xmedia Recode [9] is a free software to compress, to convert, to cut and to crop video footages.

3. MS-EXCEL is the chosen spread sheet software (data table, management, lists, sorting, functions, charts, VBA procedures to dynamically manage and control the chart and the video[10]).

4. Digital cameras are used to record 25 to $30 \mathrm{f} / \mathrm{s}$ in DVD format (780 x 512) or full HD format (1920 x 1080). A digital camera "Casio Exilim EX-FH20" to record video at $210 \mathrm{f} / \mathrm{s}$ in the format $360 \times 480$.

These softwares are updated/upgraded when there are new versions, or changed when other software allows more operational features.

Computers are running with Windows 7 or Windows 10.

The 5 applications in the course are:

1) Kinovea and MS-Excel working in two side by side windows.

2) Analysis of one or two video footages in MS-Excel using the window media player.

3) Help decision making in MS-Excel using the window media player and MS-Powerpoint.

4) Chart-video coupling.

5) Collect data in real time of the video footage in MS-Excel using the window media player.

\section{Information concerning the chart-video coupling}

The chart-video coupling concerns all sports activities; it is intradisciplinary. The first model was built in 2009. It can help us to analyze video footage of sports activities, especially shifts of supports or shifts of center of mass.

The chart-video coupling also concerns various educational disciplines, ICT and sports, ICT and physics, it is interdisciplinary.

The chart-video coupling describe below, shows an interdisciplinary approach between ICT and physics, it was built on Courty \& Kierlik's works [11]. These authors shown that in some cases, when objects fall, onepart of these objects falls faster or slower than their centers of mass. Thus, if one raises a hinged plate on a base none lets fall this plate without imparting an initial speed, the end of the board falls faster than its center of mass. It is not always possible to "see" this or these phenomena, video analysis can be a way to present and simulate this phenomenon:

- The chart-video coupling can concern ICT training (simple functions in spreadsheets and video footages) associated with physics to help to see and to understand phenomena. 
Citation: Michel CALMET (2016) Survey of the Evolution of Two Points Belonging to Two Objects Falling without Initial Speed Dynamic Chart (Dot per Dot) Video (Frame by Frame) Coupling. J Robot Mech Eng Resr 1(4): 1-9.

- The chart-video coupling will allow to compare complexity of the video and chart simplicity.

\section{Subjects}

Students of the faculty of sport sciences in L3 (bachelor) or M1 (master). They are between 18 and 21 years old, they learnt $9 \mathrm{~h}$ (L3) or $18 \mathrm{~h}$ (M1) in computers sciences at the faculty of sport sciences. Teachers / researchers using these applications.

\section{Materials}

This paper is focused on the chart-video coupling application concerning gravity; others applications run in the same way. This application requires:

1. Record a video footage, track points, export data to MS-Excel format and inlay the trajectories on the video footage.

2. Use function in spreadsheet: $=\operatorname{vlookup}()$.

3. Build a scatter chart with 4 curves.

4. Insert an activeX object (Windows Media Player) and insert the video footage inside it.

5. Include a scroll bar, set it to move the dots and insert 5 specific lines of VBA code to control the Windows Media Player and manage the chart.

6. Include two buttons respectively calling a procedure: "Play" and "Back to frame 1".

\section{Methodology}

After presenting how the chart-video coupling runs and by extension the other applications run, we will present results:

Survey on students' interest about this course

Other intradisciplinary examples in physics (Newton's pendulum; juggling)

Modeled behaviors in judo

VBA procedures are written in the tutorials provided to students and explained during the course:

1) 16 lines of VBA code to link the chart and video.

a. 6 lines to start and to stop the sub procedure (Private Sub... End Sub)

b. 5 lines to comment and explain the procedure

c. 5 specific lines of VBA code
Collect the data, present the data, simulate the experiment.

\section{Collect the data in 3 steps}

Recreate the situation of a hinged plate on a horizontal axis with a goblet and a ballpoint: The end of a hinged plate about a horizontal axis falls faster than the acceleration of gravity, if the inclination is not too great. If one fixes a goblet, and one ballpoint is placed nearby, the goblet will fall faster than the ball (which freely falls) and the goblet will collect the ballpoint as shown in Figure 1 and Figure 2.

The use of "Casio Exilim EX-FH20" at $210 \mathrm{f} / \mathrm{s}$ to record the sequence of the drop: The Casio Exilim EX-FH20 is used to record the sequence of the drop as shown in Figure 3.

The use of Kinovea software to: (i) collect the shifts of the points and superimpose their trajectories on the video; (ii) export the coordinates of the points collected in a compatible format for a spreadsheet (here xlm format). Click on the "picture $t=0.00 \mathrm{~s}$ " to
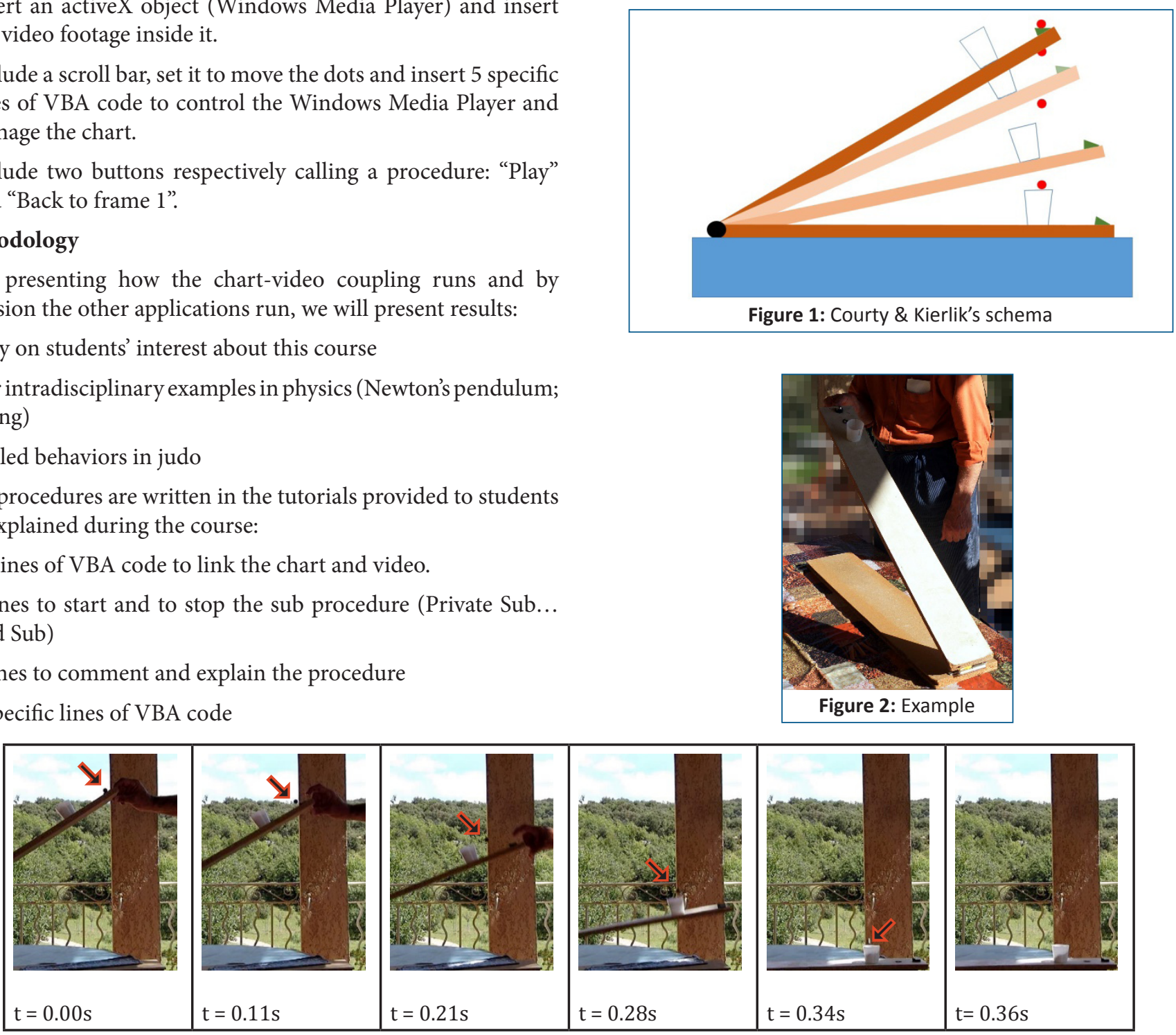

Figure 3: Chronophotography from recorded video. The red arrow indicates the position of the ballpoint 
Citation: Michel CALMET (2016) Survey of the Evolution of Two Points Belonging to Two Objects Falling without Initial Speed Dynamic Chart (Dot per Dot) Video (Frame by Frame) Coupling. J Robot Mech Eng Resr 1(4): 1-9.

see the video. It is precisely explained in Figure 4.

\section{Presenting the drop in MS Excel}

The above collection of drop presented in MS Excel by building a dynamic chart enabling a video type of animation as shown in Figure 5.

\section{Simulate with MS-Excel:}

The next step is to stipulate with MS-Excel by chart video coupling as shown in Figure 6.

VBA procedures are written in the tutorials provided to students and explained during the course:

16 lines of VBA code to link the chart and video.

a. 6 lines to start and to stop the sub procedure (Private Sub... End Sub)

b. 5 lines to comment and explain the procedure

c. 5 specific lines of VBA code

Private Sub Command Button1_Click()

'click on "Play"button to run the Windows Media Player

Feuil1.Windows MediaPlayer1.Controls.Play

End Sub

Private Sub CommandButton2_Click() “clickon "RA1" button to pause the Windows Media Player

Feuil1.WindowsMediaPlayer1.Controls.pause

' and back to frame 1

Feuil1.WindowsMediaPlayer1.Controls.currentPosition $=1 /$ Range ("P1")

' and back to value 1 in cell A1 to display the ballpoint and the goblet edge to first dot

Range (“A1") = 1

End Sub

Private Sub ScrollBar1_Change()

' Use the arrows or the scrollbar slider to calibrate the Windows Media Player on the image corresponding to the position number written in $\mathrm{A} 1$

Feuil1. WindowsMediaPlayer1. Controls.currentPosition = Range (“A1") / Range("P1")

End Sub

\section{Results}

These results concern students in sciences of sports. Students are greatly amazed at the first lesson when teacher presents applications realized previous yearby peer students. Build this

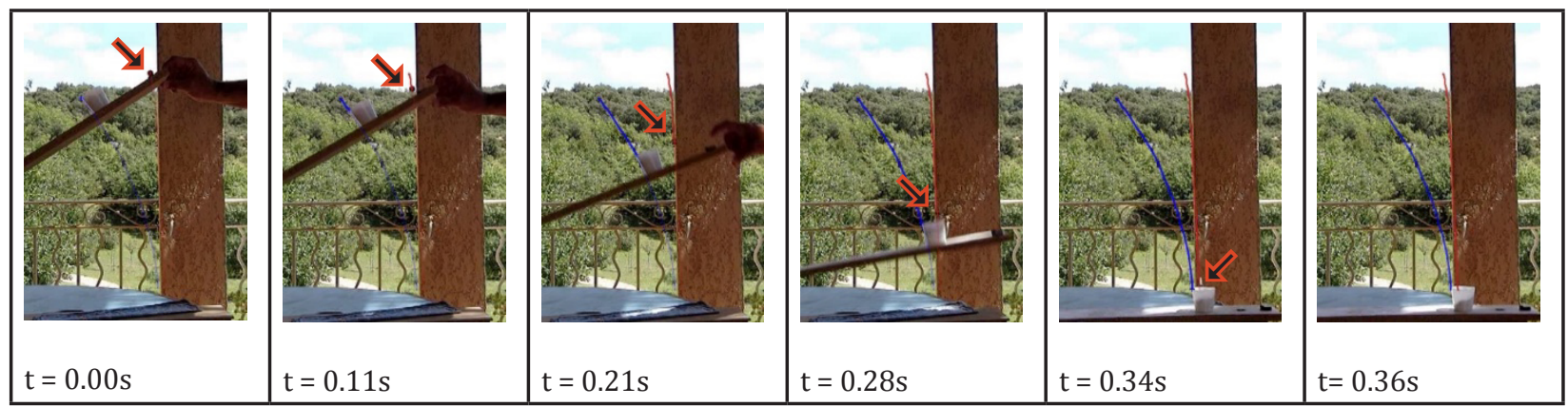

Figure 4: Chronophotography from recorded video with the trajectory of the ballpoint and the left edge of the goblet. The red arrow indicates the position of the ballpoint


Data in B3:D81 and G3:181 are obtained from Kinovea after the ballpoint tracking and the edge of the goblet tracking; and export data in spreadsheet format (Excel MS-XLM)

The columns A, E and $F$ were added, to create the image numbers $A 7: A 81$ and F7:F81

Scroll bar is linked to cell A1;

In cell A2: $=$ A1

Cells B1; $\mathrm{C} 1$ and $\mathrm{B} 2 ; \mathrm{C} 2$ calculate the coordinates of the ballpoint and the goblet edge regarding on the image number with a vlookup() function.

The chart is a scatter chart with 4 curves: Blue curvefor the goblet edge: G7: H81

Position of the goblet edge: $\mathrm{B} 2: \mathrm{C} 2$ Red curve of the ballpoint: B7: C81 Position for the ballpoint: B1: C1

Figure 5: Chart modeling. The chartbecomes "dynamic" after inserting a scroll bar to move points: "Ballpoint" (red) and "Goblet edge" (blue) 
Citation: Michel CALMET (2016) Survey of the Evolution of Two Points Belonging to Two Objects Falling without Initial Speed Dynamic Chart (Dot per Dot) Video (Frame by Frame) Coupling. J Robot Mech Eng Resr 1(4): 1-9.

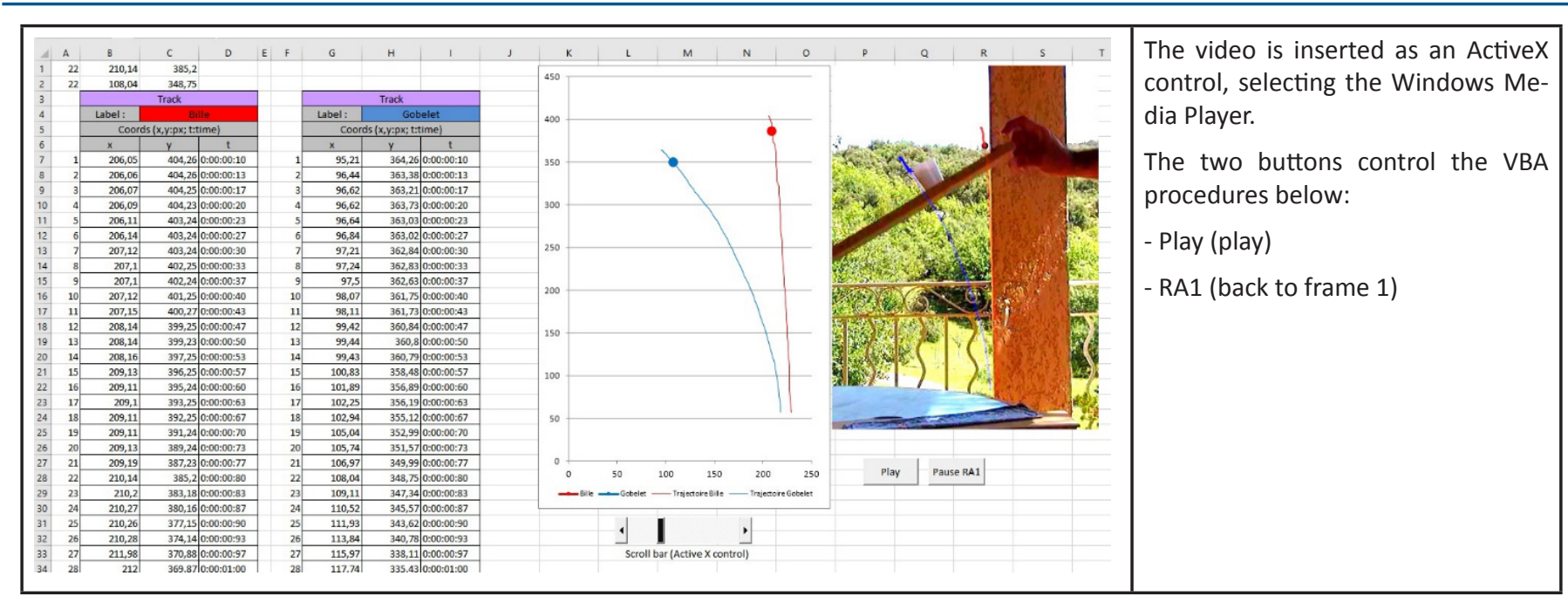

Figure 6: Simulation of the drop of the plate and the ballpoint, controlled with the scroll bar

kind of application seems them impossible, but after having read the tutorial, they understand important items of work involved. The real student work points become:

"What sport do I choose?"

"What will I show in the selected video footage"

Technology became a tool to better show a video analysis of sports.

Satisfaction surveys concerning the course, shown that the course is adapted to the curriculum; it helps to understand how run professional softwares but this course is "time consuming" (students spend a long time to organize their ICT knowledge with course objectives and video analysis). Total 168 students replied to an investigation from 2008 to 2010. Since 2010, students realized the applications and their results are always good.

The example with gravity can be extended to Newton's pendulum.

A Newton's pendulum is placed on a table. A digital camera "Casio Exilim EX-FH20" at $210 \mathrm{f} / \mathrm{s}$ is placed on the same table to record the video footage. Pendulum and digital camera are on parallel lines. A red dot paper is pasted on left ballpoint, a blue one is pasted on right ballpoint.

Two cases were studied: red left ballpoint release and blue right ballpoint release.

Ballpoint was released when user see the ballpoint curve on the rear pillar line (Figure 7 and Figure 8).

In kinovea, before tracking point, the origin of the coordinate system was the left down corner, data indicate red ordinate was 157 and blue ordinate was 151 . We analyzed the 2 videos footage, and ballpoint were considered stabilized when the variation of vertical oscillations was not above 1 (red ballpoint vertical oscillations were between 157 and 158; blue ballpoint oscillations were between 149 and 150) as shown in Figure 9 and Figure 10.

These examples can help to compare the theory (the amortization of a mechanical oscillator should be pseudo-periodically [12]) and ground truth (a rebound is observed during amortization).

In these 2 cases after stabilization, charts show 3 phases: oscillations, disturbance phase, rebound.

The example with gravity can be extended to juggling to show



Figure 7: Release of left red ballpoint

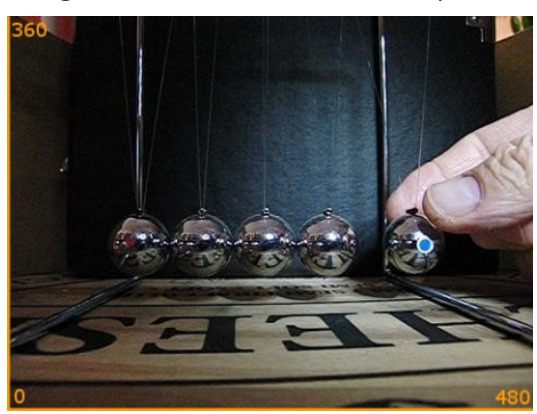

Figure 8: Release of right blue ballpoint

the regularity of the curves of the 3 balls as shown in Figure 11 and Figure 12.

\section{Modeled behaviors in judo}

The $1^{\text {st }}$ application combines kinovea and MS-Excel was used to model the judoka's system of attacks (cf. Figure 13). The two softwares are running in two side by side windows on the screen. User runs Kinovea to record key frames, after "Pause" in Kinovea, user writes 3 criteria (time, direction of attack, score) in MS-Excel. This organization does not require specific materials. The most difficult was to establish what are the criteria to collect. Two papers $[13,14]$ were published with this application. Click on the picture to see the video.

First analyzes were made in 2004/2006. Since 2010, students repeated the protocol with this organization to collect data. We presented a poster during the Judo European Championship in Montpellier in 2014.

Modeling behaviors observed in judo[15], data were collected 
Citation: Michel CALMET (2016) Survey of the Evolution of Two Points Belonging to Two Objects Falling without Initial Speed Dynamic Chart (Dot per Dot) Video (Frame by Frame) Coupling. J Robot Mech Eng Resr 1(4): 1-9.

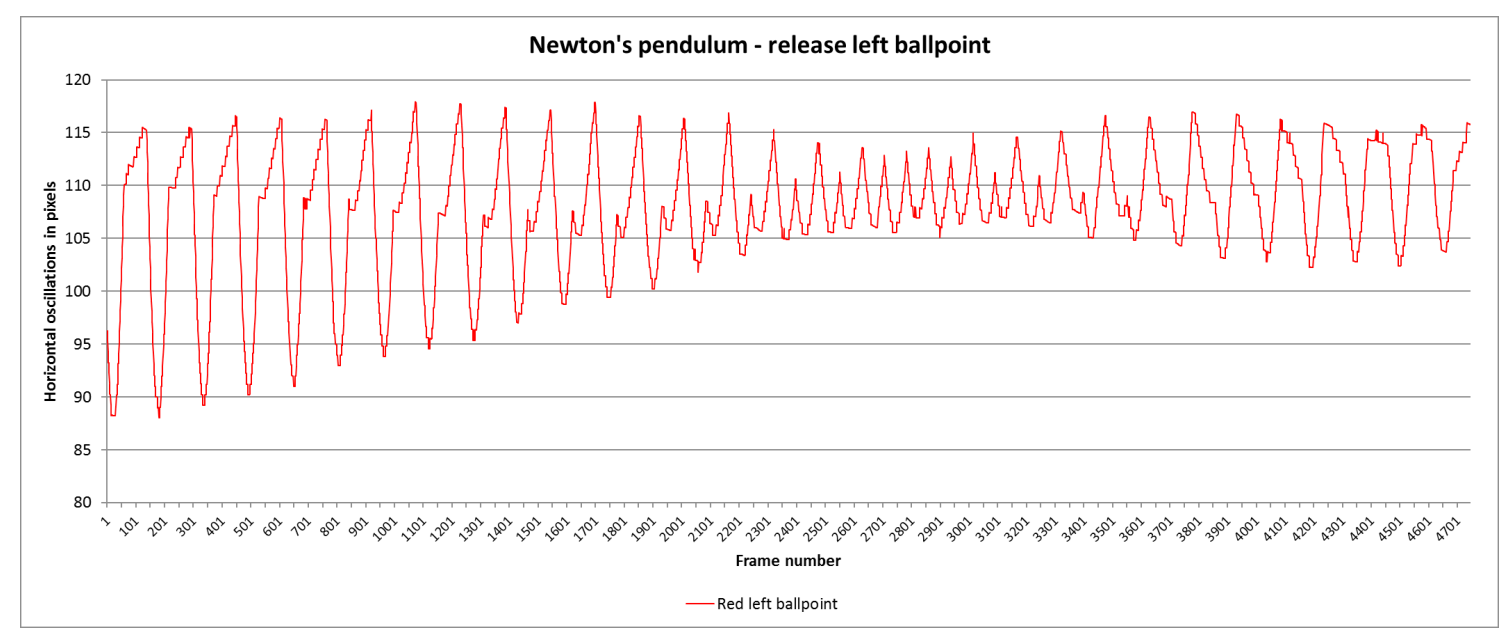

Figure 9: horizontal oscillations of the red ballpoint in Newton's pendulum

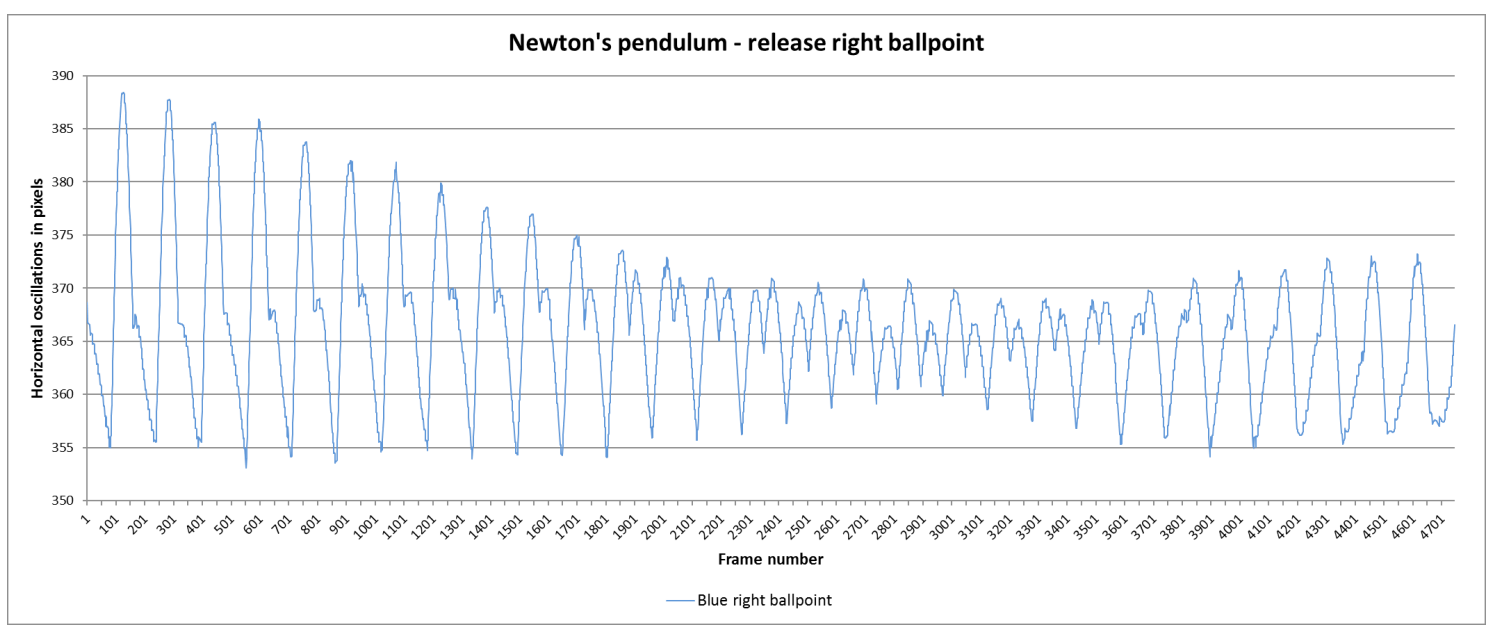

Figure 10: horizontal oscillations of the blue ballpoint in Newton's pendulum



Figure 11: Picture from application chart-video coupling with juggling video footage

with the $2 \mathrm{~d}$ application that students have to use, in which the Windows Media Player is integrated in an MS-Excel sheet.

User can note the criteria, sort, count them, process the data and display charts:

In judo, the briefness and the capacity of simultaneous actions, the multiple interactions and the result at stake result in a complex system that is most of the time clarified by the coach to the contestant. A computerized observation may help to highlight some actions associated to success from that complex system. The winners of 35 contests were analyzed ( 9 beginners, 16 intermediate levels, and 10 experts) through their behaviors (8 criteria concerning approaches and kumi-kata; cf. Figure 15).

Video footages were recorded at 25f/s, duration of experts' combat was $5 \mathrm{~min}$, the analysis was done frame by frame. Experts' 
Citation: Michel CALMET (2016) Survey of the Evolution of Two Points Belonging to Two Objects Falling without Initial Speed Dynamic Chart (Dot per Dot) Video (Frame by Frame) Coupling. J Robot Mech Eng Resr 1(4): 1-9.

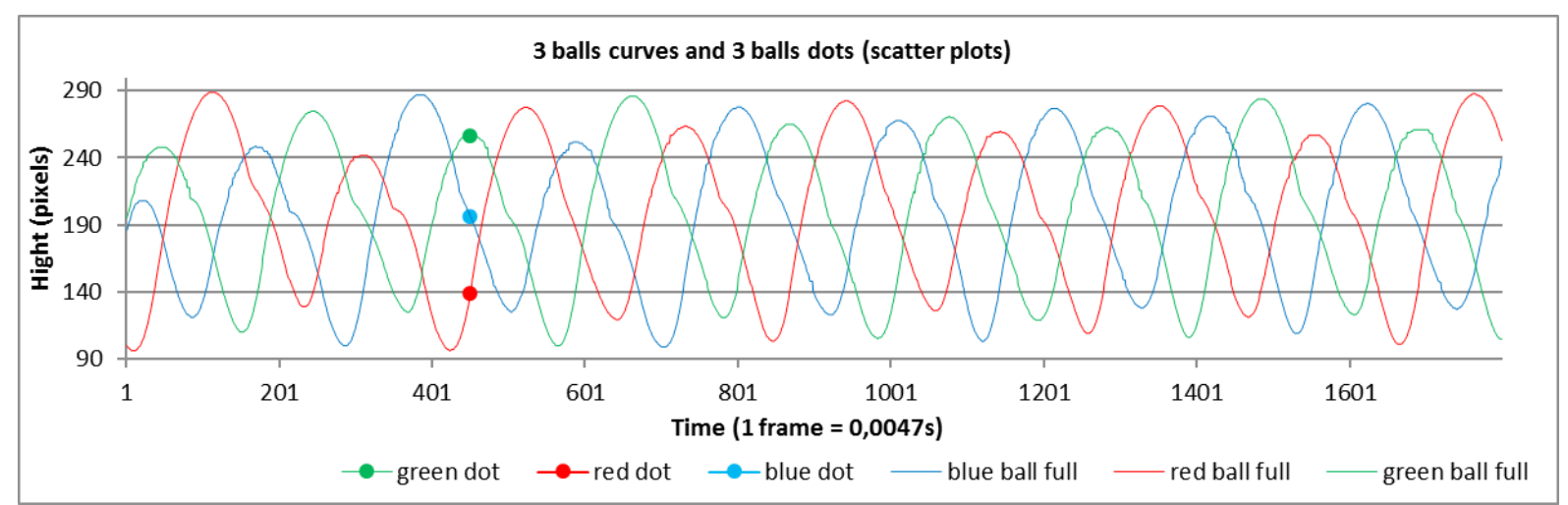

Figure 12: Curves obtained with kinovea, video footage contains 1800 frames; video was recorded at 210f/s

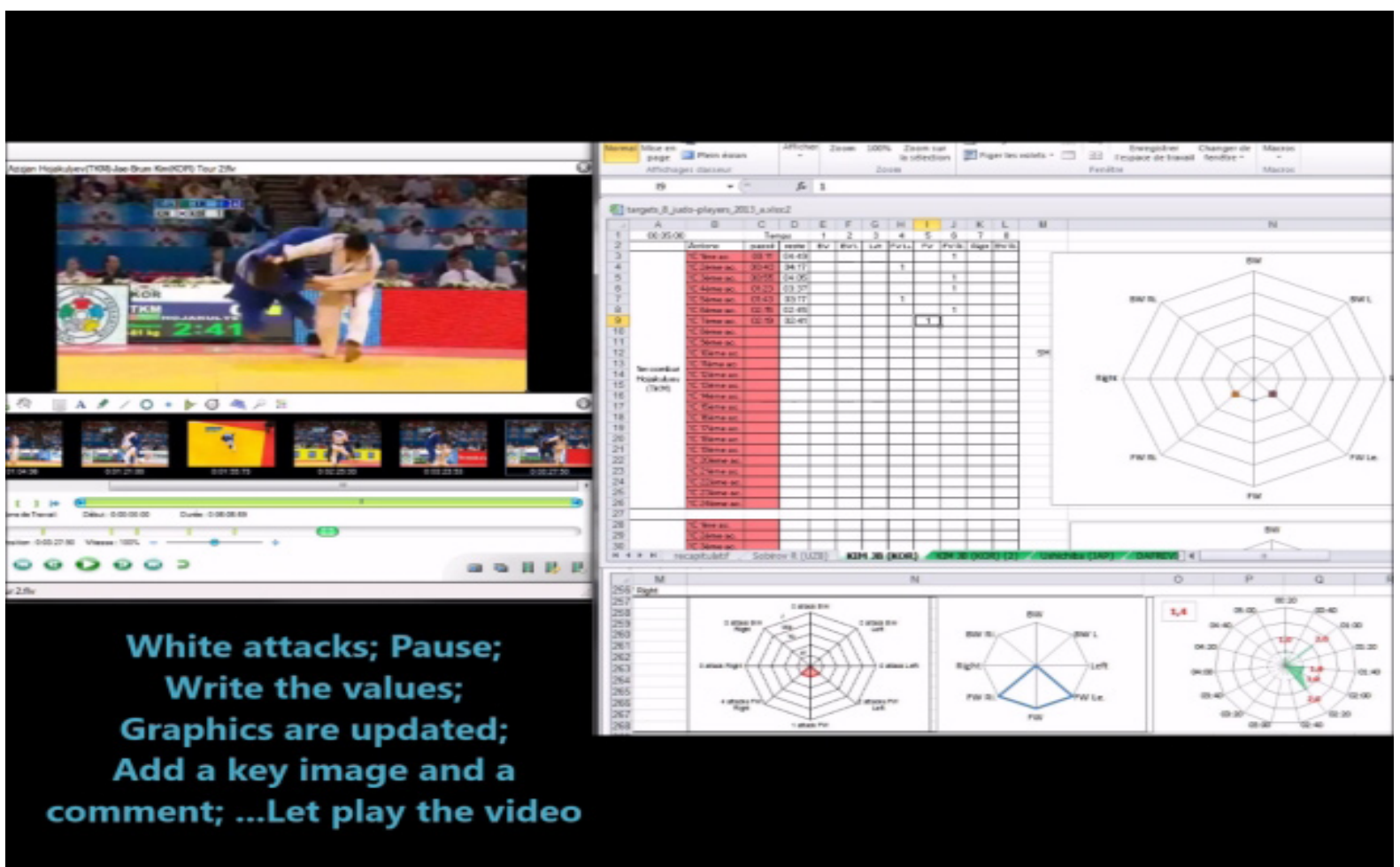

Figure 13: Kinovea and MS-Excel side by side to collect behaviors, charts are immediately updated

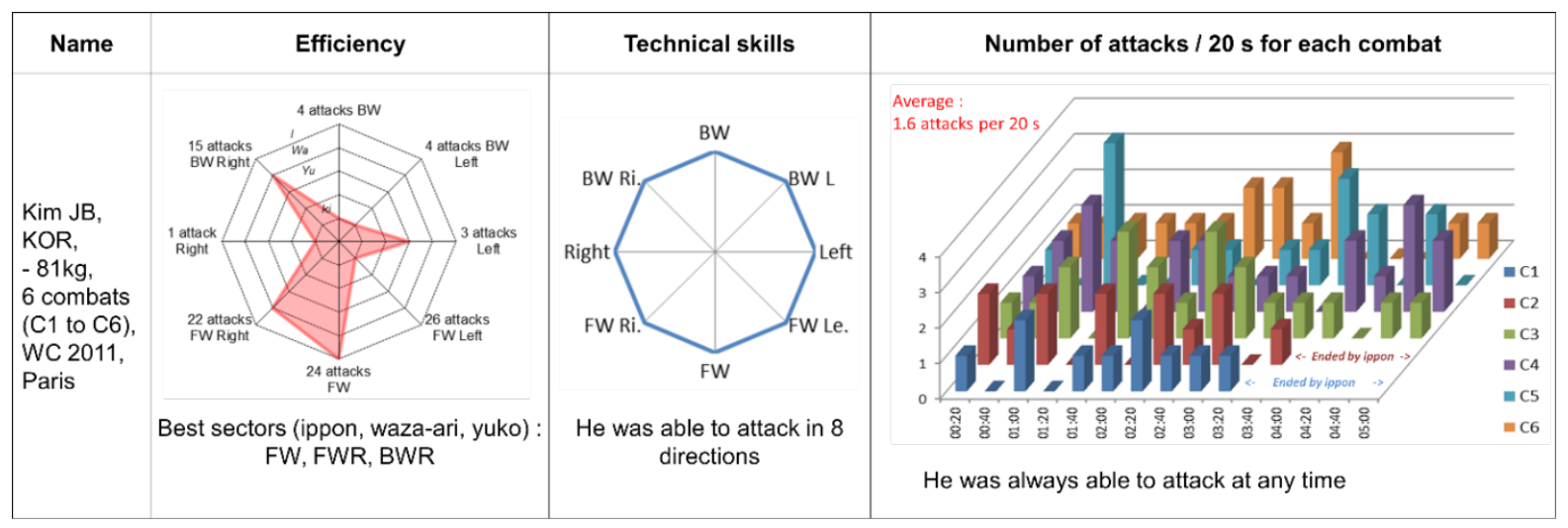

Figure 14: Chart of the system of attacks obtained using kinovea and MS-Excel

MS-excel files contain 4.500 lines (25f/s x 60s X 5min).

Grasp activity in judo (how to grasp / shift / throw) the opponent was modeled.

\section{Conclusions and perspectives}

This type of application allows:
1. Interdisciplinary and intradisciplinary training (application in physics concerning gravity was used in adult education in Algerian universities in 2014-2015).

2. Links between training and research.

3. Students develop skills in ICT and in related disciplines (sports or physical) while comprising features of professional software 
Citation: Michel CALMET (2016) Survey of the Evolution of Two Points Belonging to Two Objects Falling without Initial Speed Dynamic Chart (Dot per Dot) Video (Frame by Frame) Coupling. J Robot Mech Eng Resr 1(4): 1-9.



Figure 15: Windows Media player managed in MS-Excel, criteria, time, $\mathrm{N}^{\circ}$ frame



Figure16: Averages of profiles obtained during a combat phase

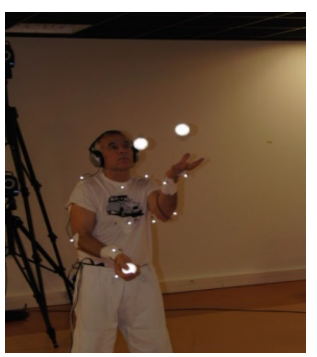

Figure 17: Juggling and video-analysis with Vicon system to collect data

video analysis.

4. New information for trainers and teachers: judoka system of attacks (Cf. Figure 14); profiles of judokas (Cf. Figure 16); juggling and video-analysis with Vicon system (Cf. Figure 17). Professional journal "L' esprit du judo" published four papers concerning system of attacks of high-level athletes in judo.

5. Simple customization to collect data (more than 65.000 columns and more than 1.000.000 lines, numerous functions to calculate and VBA to create specific procedures in MS-
Excel).

A way to prepare students to more precise works if they decide to get a PHD and to work with sophisticated materials.

Future development of the course will cover:

- Calculations of speed and acceleration, based on data collected with Kinovea.

- Statistical analyzes following the acquisition of more data from the same context.

- Build graphs in 3 dimensions (3D) with two cameras:

1. False 3D: In an orthonormal system with 3 axes $X, Y$ and $Z$, one only modifies the axis $\mathrm{Z}$ angle. The mathematical knowledge required is trigonometry studied in high school.

2. In 3D: In an orthonormal system the angles of the three axes may vary. The mathematical knowledge required are rotations of matrices; these concepts are taught in higher mathematics.

- Integration of new applications on smartphone (accelerometers, gyroscopes).

- Improve tactile applications to collect data in real time. 
Citation: Michel CALMET (2016) Survey of the Evolution of Two Points Belonging to Two Objects Falling without Initial Speed Dynamic Chart (Dot per Dot) Video (Frame by Frame) Coupling. J Robot Mech Eng Resr 1(4): 1-9.

Table 1: Satisfaction surveys concerning the course

\begin{tabular}{|c|c|c|c|c|}
\hline & 2008-2009 & $2009-2010$ & $2010-2011$ & Average \\
\hline NB students & 60 & 49 & 59 & \\
\hline Age & 22.3 & 22.15 & 22.1 & 22.2 \\
\hline $\mathrm{F}$ & 19 & 16 & 22 & \\
\hline M & 41 & 33 & 37 & \\
\hline Do you own a personal computer? (Yes) & $98.3 \%$ & $93.9 \%$ & $98.3 \%$ & $96.8 \%$ \\
\hline Do you think this course must be in the curriculum? (Yes, Rather Yes) & $85.0 \%$ & $73.5 \%$ & $94.9 \%$ & $84.5 \%$ \\
\hline Do you think this teaching organization is relevant? (Yes, Rather Yes) & $85.0 \%$ & $65.3 \%$ & $74.6 \%$ & $75.0 \%$ \\
\hline Do you perceive this course in adequation with your chosen curriculum ? (Yes, Rather Yes) & $73.3 \%$ & $65.1 \%$ & $83.1 \%$ & $73.8 \%$ \\
\hline Do you perceive course contents adapted to your knowledges? (Yes, Rather Yes) & $73.3 \%$ & $75.5 \%$ & $79.7 \%$ & $76.2 \%$ \\
\hline Are you satisfied with the progress of the course (progressive and interactivity)? (Yes, Rather Yes) & $90.0 \%$ & $75.6 \%$ & $88.1 \%$ & $84.6 \%$ \\
\hline Are you satisfied with the documents (course materials) put on line? (Yes, Rather Yes) & $91.7 \%$ & $100 \%$ & $86.4 \%$ & $92.7 \%$ \\
\hline The amount of work required outside of class hours seems ? (Too important, Important) & $68.4 \%$ & $73.4 \%$ & $52.5 \%$ & $64.9 \%$ \\
\hline
\end{tabular}

\section{References}

1. Adam HC. Eadweard Muybridge: The Complete Locomotion Photographs, Taschen. 2010.

2. Frizot M. Etienne-Jules Marey : Chronophotographe, Nathan. 2004.

3. Mannoni L. Georges Demenÿ, pionnier du cinéma Douai, Pagine Editions. 1997.

4. Marey's sphygmographe in 1882 was an "onboard" sensor to retrieve subject's pulsations.

5. La main à la pâte" operation, which was launched in 1995 by the Académie des Sciences at the initiative of Georges Charpak. Nobel Prize in Physics, 1992.

6. C2I. [cited 2016 May 21]. Available from: https://c2i.enseignementsup-recherche.gouv.fr/etudiants/les-competences-du-c2iniveau-1.

7. C2I2E. [cited 2016 May 21]. Available from: https://c2i.enseignementsup-recherche.gouv.fr/etudiants/les-competences-du-c2iniveau-2-enseignant-0.
8. Kinovea. Available from: http://www.kinovea.org/.

9. Xmedia-Recode. Available from: http://www.xmedia-recode.de/.

10. Tanguy M. Utiliser Windows Media Player en VB et VBA. [updated 2007; cited 2016 May 18].Available from: http://silkyroad.developpez.com/VBA/WindowsMediaPlayer/.

11. Courty JM, Kierlik E. Tomber plus vite qu'en chute libre. Pour la science. 2013; 434:88-90.

12. Université en ligne, Physique, Systèmes oscillants. [cited 2016 May 21]. Available from: http://uel.unisciel.fr/physique/syst_oscillants/ syst_oscillants_ch03/co/apprendre_ch3_11.html.

13. Calmet M, Ahmaidi S. Survey of the advantages obtained by judoka in competition according to their level of practice. Percept Mot Skills. 2004; 99(1):284-290.

14. Calmet M, Trezel N, Ahmaidi S. Survey of the system of attacks of regional - interregional level's judoka. Percept Mot Skills. 2006; 103(3):835-840.

15. Calmet M, Miarka B, Franchini E. Modeling of grasps in judo competition contests. International Journal of Performance Analysis in Sports. 2010; 10:229-240. 